\title{
EMISSION SPECTRA OF TERAHERTZ QUANTUM CASCADE LASER
}

\author{
A. V. Antonov,${ }^{1}$ V. I. Gavrilenko, ${ }^{1 *}$ A. V. Ikonnikov ${ }^{1}$ \\ K. V. Maremyanin, ${ }^{1}$ A. A. Lastovkin, ${ }^{1}$ \\ S. V. Morozov, ${ }^{1}$ D. V. Ushakov, ${ }^{2}$ Yu. G. Sadofyev, ${ }^{3}$ \\ and N.Samal ${ }^{3}$
}

UDC 537.9

We calculated energy levels, wave functions, and energies of radiative transitions in terahertz quantum cascade lasers based on $G a A s / A l_{0.15} G a_{0.85}$ As heterostructures. Current-voltage characteristics and current dependences of laser radiation intensity were measured, and the maximum operating temperatures reaching $85 \mathrm{~K}$ were determined. Radiation spectra of quantum cascade lasers were measured for different temperatures, and the effect of intensity "pumping" from lowfrequency modes to high-frequency modes was found to happen in the case of an increase in the current and time delay of the signal capture, which is explained by heating of the sample during a pulse of the current. Application of the lasers for registration of impurity photoconductivity signals in semiconductor heterostructures was demonstrated.

\section{INTRODUCTION}

Quantum cascade lasers are small-size high-efficiency semiconductor sources of radiation in the mid infrared and terahertz wave bands. In the mid-IR band, such devices can operate in the continous-wave $(\mathrm{CW})$ regime at room temperature, and the radiation power can exceed $1 \mathrm{~W}$ [1]. In the terahertz-wave band, the highest working temperatures achieved amount to $117 \mathrm{~K}$ in the $\mathrm{CW}$ regime [2] and $178 \mathrm{~K}$ in the pulsed-pumping regime [3]. The emission power of a quantum cascade laser in the 3-4.5 THz frequency band reaches $100 \mathrm{MW}$. However, as one passes from 3 to $1 \mathrm{THz}$, the output power and the operating temperature reduce significantly, which is connected with various physical limitations. On the side of high frequencies, the operating range of terahertz quantum cascade lasers is limited by the band of residual beams in GaAs, which serves today as the basis for all known quantum cascade lasers of this range. Therefore, comprehensive detailed research of such structures having different parameters is still topical.

This paper describes theoretical calculations of wave functions and energies of transitions of quantum cascade structures, as well as detailed studies of current-voltage (I-V) characteristics, spectra and dependences on the current of the intensity of radiation of quantum cascade terahertz lasers. The possibility of using such lasers for spectroscopy of minor admixtures in semiconductor nanostructures is demonstrated.

\section{STRUCTURES UNDER CONSIDERATION}

Quantum cascade lasers manufactured by "Trion Technology" (Tempe, Arizona, USA) were considered. Design of the structures corresponded to that described in [4], and the population inversion was

*gavr@ipm.sci-nnov.ru

${ }^{1}$ Institute of Physics of Microstructures of the Russian Academy of Sciences, Nizhny Novgorod, Russia; ${ }^{2}$ Belarusian State University, Minsk, Belarus; 3 "Trion Technology", Tempe, Arizona, USA. Translated from Izvestiya Vysshikh Uchebnykh Zavedenii, Radiofizika, Vol.52, No.7, pp.550-556, July 2009. Original article submitted June 4, 2009; accepted July 25, 2009. 
achieved due to electron tunneling from the injector to the upper working level and depopulation of the lower operating level due to emission of optical phonons. Such structures were grown by the method of molecular-beam epitaxy on a GaAs(001) substrate and consisted of recurrent (about 180 periods) sets of four quantum wells of GaAs separated by the $\mathrm{Al}_{0.15} \mathrm{Ga}_{0.85} \mathrm{As}$ barriers. The widest quantum well in which period, in each optical phonons are emitted, was doped with the density $n=1.9 \cdot 10^{16} \mathrm{~cm}^{-3}$. To reduce the loss, the structure having a thickness of about $10 \mu \mathrm{m}$ was placed in a double metal waveguide having a width of about $100 \mu \mathrm{m}$ and a length of 1-2 mm, and the cavity mirrors were formed by chippings in crystallographic direction (110). The lasers were mounted on a copper cooling conduit, which acts as the bottom contact of the structure. In this work, we study the structure of two types with differing parameters, which radiated in the regions of 3 and $4.3 \mathrm{THz}$, respectively.

\section{CALCULATIONS}

The procedure of numerical calculation of energy spectra and optical transitions in a quantum cascade laser included the following stages: determination of energy levels and wave functions, calculation of matrix elements of dipole transitions, calculation of the velocity of scattering on optical phonons and electron-electron scattering, determination of surface densities of charge carriers and Fermi's quasi-levels corresponding to energetic subzones from a system of closed balance equations [5], and calculation of amplification spectra [6]. The zone diagram, energy levels, and wave functions were calculated by solving numerically the Schrödinger equation on the basis of the transfer matrix method in the expanded Bastard model allowing for the coordinate and energetic dependence of the efficient electron mass [7]. In this case, the height of potential barriers in the conductivity zone was assumed to be equal to $\Delta E_{\mathrm{c}}=0.118 \mathrm{eV}$, and the calculated values of the effective masses of charge carriers amounted to $m^{*}=0.075 m_{0}$ for barrier levels $\mathrm{Al}_{0.15} \mathrm{Ga}_{0.85}$ As and $m^{*}=0.067 m_{0}$ for quantum wells of GaAs, where $m_{0}$ is the electron mass.

Figure $1 a$ shows the results of calculating the energy spectrum $E_{\mathrm{c}}(z)$ in the conductivity zone and squared absolute values of the electron wave functions calculated for two cascades in the electric field close to the one optimal for the laser operation, and Fig. 1b, amplification spectra for five different values of field intensity. The widths of GaAs/AlGaAs layers in the period from left to right, starting on the wide quantum well, are $16.09,3.66,9.36,4.78,8.20,2.62,6.79$, and $4.25 \mathrm{~nm}$. The carriers are injected to the upper working levels of the $(N-1)$ st cascade $(3, N-1)$ and $(2, N-1)$ by means of tunneling from level $(4, N-1)$ of the injector quantum well. Optical transitions occur between working levels $(3, N-1)$ and $(5, N)$, as well as between $(2, N-1)$ and $(1, N-1)$. Greater values of the matrix transfer elements correspond to the first pair of levels: $z_{(3, N-1),(5, N)}=4.9 \mathrm{~nm}$, whereas $z_{(2, N-1),(1, N-1)}=0.7 \mathrm{~nm}$. To ensure population inversion between the levels, the zone scheme of the cascade is chosen such as to make the energy gap between levels $(5, N)$ and $(4, N)$ and levels $(1, N-1)$ and $(3, N)$ close to the energy of the longitudinal optical phonon in GaAs. As is seen in Fig. 1b, for this design version and a specified electric field, amplification takes place at a frequency of about $3 \mathrm{THz}$, which corresponds to the distance between working levels $(3, N-1)$ and $(5, N)$, as well as $(2, N-1)$ and $(1, N-1)$.

\section{METHODOLOGY OF THE EXPERIMENT}

The measurements were performed at low temperatures $T=7-90 \mathrm{~K}$ in the pulse regime. The laser was installed in vacuum on a copper cooling conduit, in the "Displex DE-202S" closed-cycle helium cryostat. To measure the amplitude of pulses (voltage, current, emission signal, etc.), we used a two-channel strobing integrator (boxcar) manufactured by Stanford Research Systems. The laser emission was detected with an extrinsic Ge:Ga photoreceiver situated in the light-guiding module in a transport Dewar vessel. Emission spectra were recorded by a "Bruker Vertex 80v" Fourier spectrometer in the step scan regime. In the latter case, the detected emission signal was sent from the output of the strobing integrator to the ADC of the Fourier spectrometer.

The possibility of using lasers for semiconductor spectroscopy, extrinsic photoconductivity of volume 

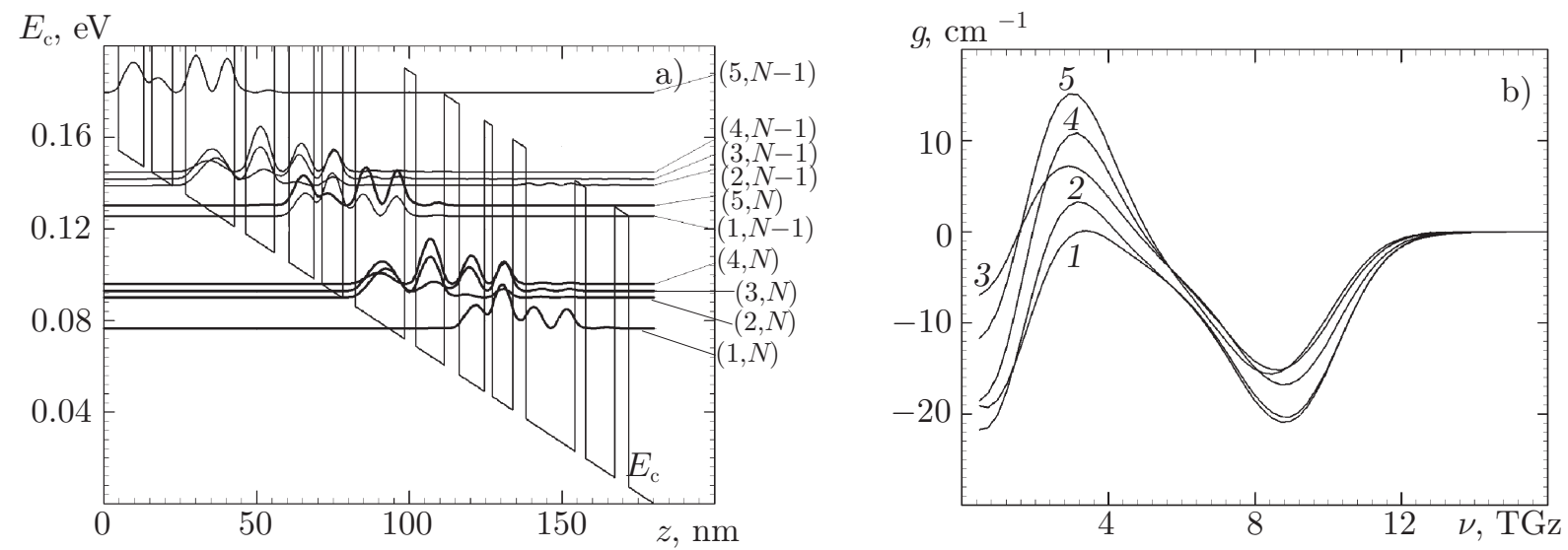

Fig. 1. Results of the calculations of the quantum cascade laser in the $3 \mathrm{THz}$ band: diagram of the conductivity zone $E_{\mathrm{c}}(z)$ and squares of the absolute values of electron wave functions in the quantum cascade structure placed in the electric field $E=8.8 \cdot 10^{5} \mathrm{~V} / \mathrm{m}(\mathrm{a})$ and amplification spectra for different values of the electric field intensity (b): $E=8.0 \cdot 10^{5} \mathrm{~V} / \mathrm{m}$ (curve 1), $8.2 \cdot 10^{5} \mathrm{~V} / \mathrm{m}$ (curve 2), $8.4 \cdot 10^{5} \mathrm{~V} / \mathrm{m}$ (curve 3), 8.6 $10^{5}$ $\mathrm{V} / \mathrm{m}$ (curve 4), and $8.8 \cdot 10^{5} \mathrm{~V} / \mathrm{m}$ (curve 5).
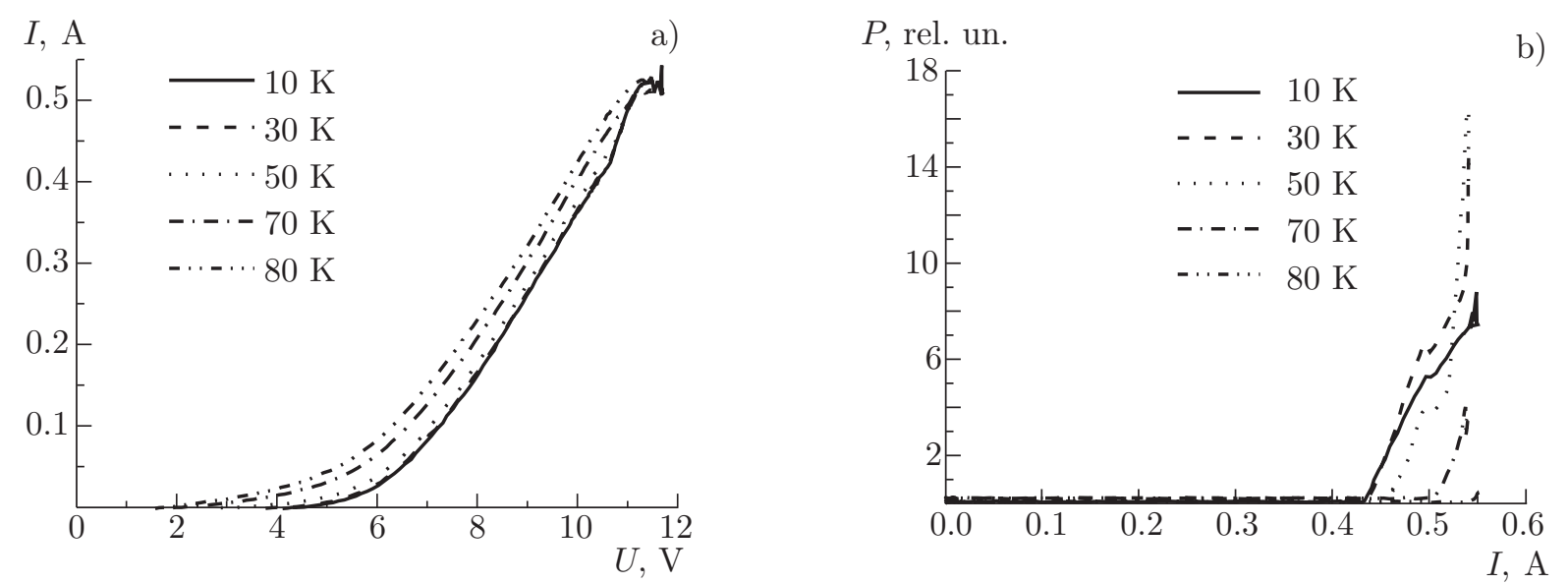

Fig. 2. Measured characteristics of the 3-THz quantum cascade laser at different temperatures: I-A characteristic (a) and current dependences of the integral emission intensivity $(b)$.

n-GaAs material and the p-Ge/GeSi heterostructure was studied. Terahertz emission of the laser was sent via a light guide to the cryostat with a superconducting magnet, at the center of which the studied sample was installed.

\section{EXPERIMENTAL RESULTS AND DISCUSSION}

Typical I-V characteristics of the $3-\mathrm{THz}$ quantum cascade laser are shown in Fig. $2 a$. It is seen that a noticeable current arises in this laser at low temperatures for an applied voltage of over $4 \mathrm{~V}$, which corresponds to alignment of levels ensuring the passage of the current through the cascade of the structure at the cost of injection to levels $(5, N)$ and $(1, N-1)$ (see Fig. 1 ). As the temperature increases up to $80 \mathrm{~K}$, the current growth starts at an applied voltage of about $2 \mathrm{~V}$ already, which is probably due to thermal activation of the carriers to the above-lying levels. Starting at a voltage of $6 \mathrm{~V}$, an interval of linear current growth starts at all temperatures. This interval is connected with the currents' growing through the structure due to injection to the upper working level $(3, N-1)$. Finally, at the end of the linear interval (at a voltage of about $10 \mathrm{~V}$ ) a sharp break in the $\mathrm{I}-\mathrm{V}$ curve is observed, which corresponds to the onset of laser emission. It is natural to associate it with a decrease in the lifetime of the carriers at the upper working level due 
to stimulated emission of photons. As the voltage increases further, one observes saturation of (and even some decrease in) the current, which results from a mismatch of the injector level and upper working level $(3, N-1)$.

Figure $2 b$ shows current dependences of the integral emission intensity at different temperatures for the $3 \mathrm{THz}$ quantum cascade laser. At low temperatures, laser emission started at a current of $0.46 \mathrm{~A}$, which corresponds specifically to the break in the I-V curve (see Fig. $2 a$ ). At temperatures up to $50 \mathrm{~K}$, one could see a peculiarity in these dependences for a current of about $0.5 \mathrm{~A}$, after which an interval of faster growth of the emission intensity was observed. Most probably, it was caused by changes in the mode content of the radiation. When the maximum value of the current, which corresponded to the beginning of the region of negative differential conductivity, was reached, a sharp decrease in the emission intensity was observed. Emission properties of the laser did not undergo any significant changes at up to a temperature of $50 \mathrm{~K}$, after which the emission intensity drastically decreased, which was connected, primarily, with a decrease in the electron lifetime at the upper working level due to a higher frequency of electron scattering by acoustic phonons. The maximum operating temperature of this laser type amounted to about $80 \mathrm{~K}$. The obtained values of the critical current and the maximum temperature are comparable with the best values presented in literature for $3 \mathrm{THz}$ lasers with double metal waveguides [2].

Figure 3 shows emission spectra for both types of the lasers under consideration. For the $3 \mathrm{THz}$ laser (Fig. $3 a$ ), the spectra were recorded with limiting resolution of the spectrometer equal to $0.2 \mathrm{~cm}^{-1}$. It is easily seen that at a low temperature $(7 \mathrm{~K})$ and a laser voltage of $14.6 \mathrm{~V}$, only one line with a maximum of $99.6 \mathrm{~cm}^{-1}(2.99 \mathrm{THz})$ is present in the spectrum, which agrees well with the calculation results. At a higher laser voltage $(14.9 \mathrm{~V})$, a few more lines shifted into the region of higher energies are observed in the emission spectrum at the same temperature. The highest-power lines are situated equidistantly, and the distance between them, probably, corresponds to the distance between the longitudinal modes of the cavity. We relate the shift of the generation band into the short-wave region to an increase in the working-region temperature, which is caused by an increase in the voltage and current. This is confirmed by the studies of the emission spectra at different temperatures. As is seen in Fig. $3 a$, at a temperature of $50 \mathrm{~K}$ the main spectrum line also shifts towards the greater energies.

In most detail, the effect of emission spectrum detuning with heating into the high-frequency region has been traced for the $4.3 \mathrm{THz}$. Figure $3 b$ shows emission spectra for different temperatures at the identical current via the laser, namely, $1.4 \mathrm{THz}$. One can see the presence of two main spectral lines at frequencies near 144 and $145.5 \mathrm{~cm}^{-1}$, which corresponds to $4.3 \mathrm{THz}$. As the temperature increases, the "pumping" of the intensity from the low-frequency to a higher-frequency mode is observed along with the decrease in the
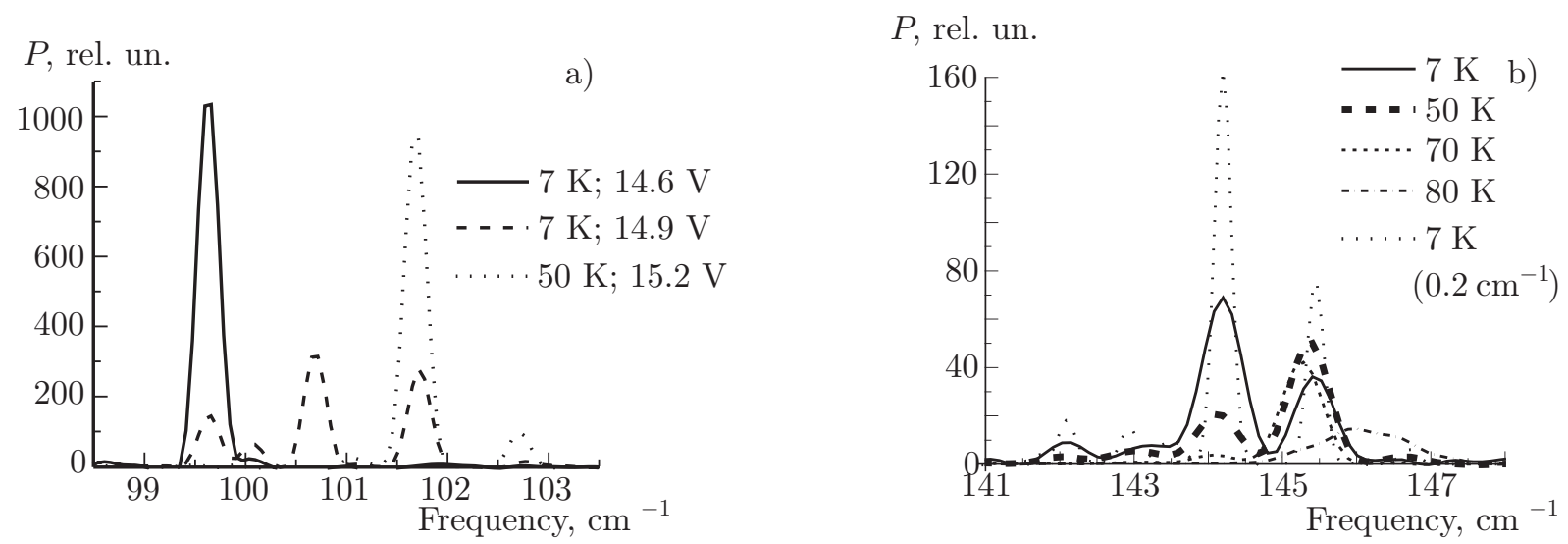

Fig. 3. Emission spectra of quantum cascade lasers for the $3 \mathrm{THz}(a)$ and $4.3 \mathrm{THz}(b)$ bands. For the first laser, the spectra were measured with a resolution of $0.2 \mathrm{~cm}^{-1}$ and for the second, of $0.5 \mathrm{~cm}^{-1}$ (except for the last spectrum). In the second case, the spectra were recorded for one and the same current through the laser, which amounted to $1.4 \mathrm{~A}$. 

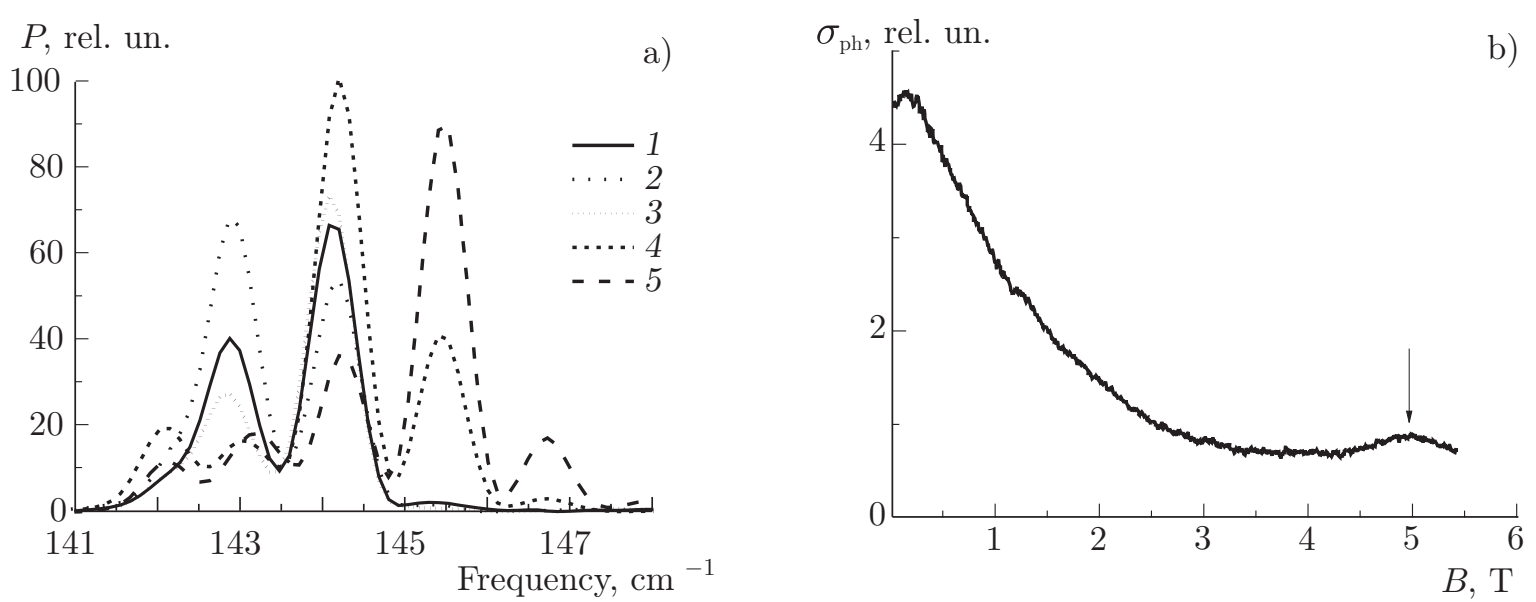

Fig. 4. (a) Emission spectra of the $4.3 \mathrm{THz}$ laser, which were measured with a resolution of $0.5 \mathrm{~cm}^{-1}$ at a temperature of $7 \mathrm{~K}$ and various positions (with respect to time) of the sampling pulse (strobe) relative the emission pulse for three values of the applied voltage: curve 1 corresponds to $14.4 \mathrm{~V}$ (entire pulse), 2 to $14.4 \mathrm{~V}$ (beginning of the pulse), 3 to $14.4 \mathrm{~V}$ (end of the pulse), 4 to $15.3 \mathrm{~V}$ (beginning of the pulse), and 5 to $15.8 \mathrm{~V}$ (beginning of the pulse). (b) Dependence of the extrinsic photoconductivity of the heterostructure $p$-Ge/GeSi, which was measured by the $3 \mathrm{THz}$ quantum cascade laser, on the magnetic field.

radiation intensity. This effect can be explained by both the decrease in the amplification, and the increased absorption of the electromagnetic radiation in the free-carrier laser, which grows, as is known, in direct proportion with the squared wavelength. In combination, the result of the two factors is that the maximum of the amplification coefficient should shift toward the region of higher frequencies, which is observed for the both types of the lasers.

In the paper, the emission spectra of the $4.3 \mathrm{THz}$ laser were also obtained for different values of the applied voltage and at different time moments during the laser emission pulse (Fig. 4a). The first three curves were obtained for a voltage of $14.4 \mathrm{~V}$. The first curve is the emission spectrum averaged over the greater part of the emission pulse with a duration of about $10 \mu \mathrm{s}$ (which corresponds to the conditions of spectrum measurements in Fig. 3b). For this purpose, the duration of the sampling at the strobing integrator was chosen almost equal to the duration of the emission pulse. The second and third spectra were measured for thrice as short strobe duration. Here, the second spectrum corresponds to the initial part of the emission pulse, and the third spectrum, to the pulse end. It is easily seen that the "integral" spectrum is essentially the superposition of the second and third spectra. From comparison of the latter, one can see that by the end of the radiation pulse, the intensity is "pumped" through the low-frequency into the high-frequency mode, which can be connected with the heating of the active region during the emission pulse. An increase in the current through the laser leads to a similar effect. The second, fourth, and fifth spectra are measured at the initial moment of the radiation pulse for laser voltages $14.4,15.3$, and $15.8 \mathrm{~V}$, respectively. One can see that as the voltage increases, the intensity of high-frequency modes grows progressively and the intensity of low-frequency modes decreases, which is also most probably connected with laser heating. Another possible reason for the high-frequency shift of the spectrum during the current increase and, therefore, of the electric field in the structure is the increase in the distance between the working levels (Fig. 1a) "related" to the neighboring quantum wells, due to a greater drop of the voltage at the barrier which separates them. As a result, the frequency of the working transition grows correspondingly, which leads to a shift of the amplification line and the spectrum of laser emission.

To demonstrate the possibility of using lasers for spectroscopy tasks, a thick epitaxial film of n-GaAs and the p-Ge/GeSi \#306a heterostructure were used (see [8]). In both cases, resonances connected with the transitions between the main and excited states of minor admixtures were observed during scanning of the magnetic field. For the p-Ge/GeSi heterostructure, the position of the peak detected in the region of 
$5 \mathrm{~T}$ (Fig. 4b) corresponds well to the extrinsic transition $1 s \rightarrow 2 p^{+}$, which was observed earlier [8], for small acceptors at the center of the Ge quantum well.

This work was supported by the Belarus Republic Foundation for Basic Research, the Russian Foundation for Basic Research (projects F08R-119 and 08-02-90054, 08-02-00962), and the Russian Academy of Sciences.

\section{REFERENCES}

1. Y. Bai, S. R. Darvish, S. Slivken, et al., Appl. Phys. Lett., 92, 101105 (2008).

2. B. S. Williams, Nature Photonics, 1, 517 (2007).

3. M. A. Belkin, J. A. Fan, S. Hormoz, et al., Optics Express, 16, 3242 (2008).

4. B. S. Williams, H. Callebaut, S. Kumar, et al., Appl. Phys. Lett., 82, 1015 (2003).

5. D. V. Ushakov and I. S. Manak, Optika i Spektroskopia [in Russian], 104, 846 (2008).

6. D. V. Ushakov and I. S. Manak, Journ. Applied Spectrosc. [in Rusian], 74, 801 (2007).

7. K. H. Yoo, L. R. Ram-Mohan, and D. F. Nelson, Phys. Rev. B, 39, 12808 (1989).

8. V. I. Gavrilenko, I. V. Erofeeva, A. L. Korotkov, et al., JETP Lett., 65, 209 (1997). 